\title{
PROJECTION LATERAL BANDS AND LATERAL RETRACTS
}

\author{
KAMIŃSKA A. ${ }^{1}$, KRASIKOVA I. ${ }^{2}$, POPOV M. ${ }^{1,3}$
}

\begin{abstract}
A projection lateral band $G$ in a Riesz space $E$ is defined to be a lateral band which is the image of an orthogonally additive projection $Q: E \rightarrow E$ possessing the property that $Q(x)$ is a fragment of $x$ for all $x \in E$, called a lateral retraction of $E$ onto $G$ (which is then proved to be unique). We investigate properties of lateral retracts, that are, images of lateral retractions, and describe lateral retractions onto principal projection lateral bands (i.e. lateral bands generated by single elements) in a Riesz space with the principal projection property. Moreover, we prove that every lateral retract is a lateral band, and every lateral band in a Dedekind complete Riesz space is a projection lateral band.

Key words and phrases: orthogonally additive operator, Riesz space, lateral band.
\end{abstract}

\footnotetext{
${ }^{1}$ Institute of Exact and Technical Sciences, Pomeranian University in Słupsk, 76-200, Słupsk, Poland

2 Zaporizhzhya National University, 66 Zukovs'koho str., 69600, Zaporizhzhya, Ukraine

${ }^{3}$ Vasyl Stefanyk Precarpathian National University, 57 Shevchenka str., 76018, Ivano-Frankivsk, Ukraine

E-mail: anna.kaminska@apsl .edu.pl (Kamińska A.), irynazpukregmail .com (Krasikova I.), misham. popov@gmail.com (Popov M.)
}

\section{INTRODUCTION}

Recent development of the theory of orthogonally additive operators on Riesz spaces brought appearance and study of new specific notions like lateral order, lateral ideal and lateral band in a Riesz space. These notions have appeared to be so important for the study of orthogonally additive operators, as the corresponding notions of ideal and band are important for linear operators. In the present paper, we find conditions on a Riesz space under which every lateral band is a projection lateral band. We also find formulas for lateral band projections.

In this paper, we familiarly use terminology and notation on Riesz spaces from textbook [1]. The specific notation $x=\bigsqcup_{i \in I} x_{i}$ is used to express that $x$ is a disjoint sum of $x_{i}$, that is, $x=\sum_{i \in I} x_{i}$ and $x_{i} \perp x_{j}$ if $i \neq j$. The notation $x \sqsubseteq y$ means that $x$ is a fragment ${ }^{1}$ of $y$, that is, $x \perp(y-x)$ for elements of a Riesz space $E$. Given any $e \in E$, we set $\mathfrak{F}_{e}=\{x \in E: x \sqsubseteq e\}$. If a Riesz space $E$ has the principal projection property, then for every $e \in E \backslash\{0\}$ by $P_{e}$ we denote the order projection onto the band generated by $\{e\}$.

$\overline{\mathrm{y} \Delta \mathrm{K} 517.982}$

2010 Mathematics Subject Classification: Primary 46A40; Secondary 47B65.

${ }^{1}$ component, in the terminology of [1] 


\subsection{Orthogonally additive operators}

In pioneer papers [4,5], J.M. Mazón and S. Segura de León introduced, studied and discovered main vector lattice properties of orthogonally additive operators. Recall that a map $T: E \rightarrow F$ between Riesz spaces $E$ and $F$ is called an orthogonally additive operator if $T(x \sqcup y)=$ $T(x)+T(y)$ for all disjoint pairs $x, y \in E$. An orthogonally additive operator $T: E \rightarrow F$ is said to be positive (write $T \geq 0$ ) provided $T(x) \geq 0$ for all $x \in E$ (not only for $x \geq 0$ as is claimed for positivity of linear operators). An order bounded orthogonally additive operator is called an abstract Uryson operator, and the set of all abstract Uryson operators $T: E \rightarrow F$ is denoted by $\mathcal{U}(E, F)$. The vector space $\mathcal{U}(E, F)$ is a Riesz space with respect to the order $S \leq T$ if and only if $T-S \geq 0$. Moreover, $\mathcal{U}(E, F)$ is Dedekind complete, once $F$ is [4, Theorem 3.2]. Simple examples of nonlinear abstract Uryson operators are: $T_{1}(x)=x^{+}, T_{2}(x)=|x|$ from $E$ to $E$; more general, any order bounded linear operator $T: E \rightarrow F$ defines a positive abstract Uryson operator by $S(x)=T|x|$ for all $x \in E$. For further examples (e.g., integral Uryson operators) see $[4,5,7]$.

\subsection{Lateral order, lateral ideals and lateral bands}

It is well known that ideals and bands of a Riesz space are very important for the theory of order bounded linear operators on Riesz spaces. Similarly, lateral ideals and lateral bands play an important role in the theory of abstract Uryson operators. First these notions were introduced and studied in [3], and later in [8,9] and other papers by M. Pliev, M. Popov, K. Ramdane and some other authors.

The relation $\sqsubseteq$ is a partial order on a Riesz space $E$ [8], which obviously coincides with the usual order $\leq$ on the positive cone $E^{+}$. A net in $E$ which is (non-strictly) increasing with respect to the lateral order is said to be laterally increasing. A subset $G$ of $E$ is said to be laterally closed, provided for every laterally increasing net $\left(x_{\alpha}\right)$ in $G$ the existence of a supremum $x=\sqsubseteq$-sup $x_{\alpha} x_{\alpha}$ of $\left(x_{\alpha}\right)$ with respect to the lateral order in $E$ implies $x \in G$.

A subset $G$ of a Riesz space $E$ is said to be laterally solid, if for every $x \in E$ and $g \in G$ the condition $x \sqsubseteq g$ implies $x \in G$ (in other words, provided $\mathfrak{F}_{g} \subseteq G$ for all $g \in G$ ). A laterally solid subset $G$ of $E$ is called a lateral ideal of $E$, if for every $x, y \in G$ the condition $x \perp y$ implies $x+y \in G$. For example, the kernel of every positive abstract Uryson operator is a lateral ideal [8]. A laterally closed lateral ideal $G$ of $E$ is called a lateral band of $E$. Obviously, every ideal is a lateral ideal and every band is a lateral band.

Theorem 1 ([3,8]). Let $E$ and $F$ be Riesz spaces with $F$ Dedekind complete, $D$ a lateral ideal of $E$ and $T: D \rightarrow F^{+}$an order bounded orthogonally additive operator. Then the function $\widetilde{T}: E \rightarrow F$ defined by

$$
\widetilde{T} x=\sup \left\{T y: y \in \mathcal{F}_{x} \cap D\right\}
$$

for all $x \in E$ is a positive abstract Uryson operator extending $T$. Moreover, $\widetilde{T}$ is the minimal positive abstract Uryson extension of $T$ with respect to the order on $\mathcal{U}(E, F)$.

If, moreover, $D$ is a lateral band of $E$ then the minimal extension $\widetilde{T}$ defined by (1), inherits some of compactness-like properties of $T$, e.g. AM-compactness, $C$-compactness, narrowness, strict narrowness [8, Theorems 3,4]. 


\subsection{Orthogonally additive projections onto lateral bands}

Observe that the intersection of any family of lateral ideals (respectively, lateral bands) of a Riesz space $E$ is a lateral ideal (respectively, lateral band). Hence, given any subset $A$ of $E$, there exists a minimal lateral ideal $\mathcal{I}(A)$ (respectively, a minimal lateral band $\mathcal{B}(A)$ ) including $A$, called the lateral ideal (respectively, the lateral band) generated by $A$.

Following notation of [3], we use the symbols in bold $\cup, \cup$ (respectively, $\cap, \cap$ ) to denote the lateral supremum (respectively, lateral infimum) of subsets of a Riesz space, that are, supremum and infimum with respect to the lateral order $\sqsubseteq$. A net $\left(x_{\alpha}\right)$ in a vector lattice up-laterally converges to an element $x \in E$ if $x_{\alpha} \sqsubseteq x_{\beta} \sqsubseteq x$ for all indices $\alpha \leq \beta$ and $\left(x_{\alpha}\right)$ order converges to $x$. In this case we write $x_{\alpha} \stackrel{\ell \uparrow}{\longrightarrow} x$. Obviously, $x_{\alpha} \stackrel{\ell \uparrow}{\longrightarrow} x$ holds if and only if $\left(x_{\alpha}\right)$ is laterally increasing and $\bigcup_{\alpha} x_{\alpha}=x$. A function $f: E \rightarrow F$ between vector lattices is called up-laterally continuous at a point $x \in E$ if for every net $\left(x_{\alpha}\right)$ in $E$ with $x_{\alpha} \stackrel{\ell \uparrow}{\longrightarrow} x$ in $E$ one has $f\left(x_{\alpha}\right) \stackrel{\ell \uparrow}{\longrightarrow} f(x)$ in $F$. A function $f$ is said to be up-laterally continuous provided $f$ is up-laterally continuous at every point $x \in E$.

Theorem 2 ([3]). Let $B$ be a lateral band of a Dedekind complete vector lattice $E$. Then the function $Q_{B}: E \rightarrow E$ defined by setting for every $x \in E$

$$
Q_{B}(x)=\bigcup\left(\mathfrak{F}_{x} \cap B\right),
$$

is a disjointness preserving up-laterally continuous projection of $E$ onto $B$.

A direct consequence of Theorem 2 is the following result.

Corollary 1. Let $E$ be a Dedekind complete vector lattice and $e \in E$. Then the function $Q_{e}: E \rightarrow E$ defined by setting for every $x \in E$

$$
Q_{e}(x)=\bigcup\left(\mathfrak{F}_{x} \cap \mathfrak{F}_{e}\right),
$$

is a disjointness preserving up-laterally continuous projection of $E$ onto $\mathfrak{F}_{e}$.

In Section 3 we extend Corollary 2 to the more general setting of Riesz spaces with the principal projection property by proving for every $e \in E$ the existence of a lateral band projection $Q_{e}$ of $E$ onto the principal lateral band $\mathfrak{F}_{e}$ with a more exact formula $Q_{e}(x)=x \cap e$ for all $x \in E$. Moreover, we prove the orthogonal additivity of $Q_{e}$.

\section{LATERAL RETRACTIONS}

In this section, we define lateral retractions and lateral retracts in a natural way. Then we show that lateral retracts are lateral bands, and in a Dedekind complete Riesz space the converse is also true.

Definition 1. Let $E$ be a Riesz space. A function $f: E \rightarrow E$ is called a lateral contraction if $f(x) \sqsubseteq x$ holds true for all $x \in E$.

A lateral contraction has the following obvious property.

Proposition 1. Let $E$ be a Riesz space. Then every lateral contraction $f: E \rightarrow E$ preserves disjointness, that is, for every $x, y \in E$ the condition $x \perp y$ implies $f(x) \perp f(y)$. 
Proof. Observe that for every $u, v \in E$, if $u \sqsubseteq v$ then $|u| \leq|v|$. Indeed, $u \perp(v-u)$ implies $v=u \sqcup(v-u)$ and hence $|v|=|u|+|v-u|$, which yields $|u| \leq|v|$. So, let $x, y \in E$ with $x \perp y$. Then

$$
|f(x)| \wedge|f(y)| \leq|x| \wedge|y|=0
$$

Definition 2. Let $E$ be a Riesz space. A function $f: E \rightarrow E$ is called a lateral retraction if the following conditions hold:

(i) $f$ is a projection, that is, $f^{2}=f$;

(ii) $f$ is an orthogonally additive operator;

(iii) $f$ is a lateral contraction.

A subset $A$ of $E$ is called a lateral retract if $A$ is the image of a lateral retraction $f: E \rightarrow E$.

Definition 3. A lateral band $A$ of a Riesz space $E$, which is a lateral retract, is called a projection lateral band, and the lateral retraction of $E$ onto $A$ is called the lateral band projection of $E$ onto $A$.

Remark 1. Observe that the projection $Q_{B}$ in Theorem 2 is obviously a lateral contraction. Hence, $Q_{B}$ is a lateral retraction and so, every lateral band in a Dedekind complete Riesz space is a projection lateral band.

Theorem 3. Let E be a Riesz space. Then the following assertions hold.

1. Let $A$ be a lateral retract in $E$. Then there exists a unique retraction of $E$ onto $A$.

2. Every lateral retraction is up-laterally continuous.

3. Every lateral retract in $E$ is a lateral band.

4. If, moreover, $E$ is Dedekind complete then a subset $A$ of $E$ is a lateral retract if and only if $A$ is a projection lateral band.

We need the following elementary property of orthogonally additive operators (see [6, Theorem 4.9] for more details).

Lemma 1. Let $E, F$ be Riesz spaces and $T: E \rightarrow F$ a disjointness preserving orthogonally additive operator. Then $T$ is lateral order preserving, that is, for every $x, y \in E$ the relation $x \sqsubseteq y$ implies $T(x) \sqsubseteq T(y)$.

Proof. Let $x, y \in E$ and $x \sqsubseteq y$. Since $x \perp(y-x)$, one has $T(x) \perp T(y-x)$. On the other hand, $y=x \sqcup(y-x)$ implies $T(y)=T(x)+T(y-x)$, that is, $T(y-x)=T(y)-T(x)$. Hence, $T(x) \perp T(y)-T(x)$, that is, $T(x) \sqsubseteq T(y)$. 
Proof of Theorem 3. (1) Let $f, g: E \rightarrow E$ be lateral retractions of $E$ onto $A$ and $x \in E$. By Lemma $1, f(x) \sqsubseteq x$ implies $f(x)=g(f(x)) \sqsubseteq g(x)$ and similarly $g(x) \sqsubseteq f(x)$, which yield $f(x)=g(x)$.

(2) Let $f: E \rightarrow E$ be a lateral retraction. Let $\left(x_{\alpha}\right)$ be a net in $\mathfrak{F}_{x}$ with $x_{\alpha} \stackrel{\ell \uparrow}{\longrightarrow} x$. By Lemma 1, $f\left(x_{\alpha}\right) \sqsubseteq f\left(x_{\beta}\right) \sqsubseteq f(x)$ for $\alpha \leq \beta$. The equality $x=x_{\alpha} \sqcup\left(x-x_{\alpha}\right)$ implies

$$
f(x)=f\left(x_{\alpha}\right)+f\left(x-x_{\alpha}\right) .
$$

Hence $f(x)-f\left(x_{\alpha}\right)=f\left(x-x_{\alpha}\right) \sqsubseteq x-x_{\alpha}$ and therefore ${ }^{2}\left|f(x)-f\left(x_{\alpha}\right)\right| \leq\left|x-x_{\alpha}\right|$, which yields $f\left(x_{\alpha}\right) \stackrel{o}{\longrightarrow} f(x)$. So, $f\left(x_{\alpha}\right) \stackrel{\ell \uparrow}{\longrightarrow} f(x)$ is proved.

(3) Let $A \subseteq E$ be a lateral retract and $f: E \rightarrow E$ be a lateral retraction of $E$ onto $A$. First we prove that $A$ is laterally solid. Let $x \in A$ and $y \sqsubseteq x$; our goal is to prove that $y \in A$. Since $f(y) \sqsubseteq y$, one has $(y-f(y)) \sqsubseteq y$, which yields $|y-f(y)| \leq|y|$. Similarly, $f(x-y) \sqsubseteq(x-y)$ yields $|x-y-f(x-y)| \leq|x-y|$. Hence,

$$
|y-f(y)| \wedge|x-y-f(x-y)| \leq|y| \wedge|x-y|=0
$$

and therefore

$$
0=|y-f(y)+(x-y)-f(x-y)|=|y-f(y)|+|(x-y)-f(x-y)|,
$$

which implies $y=f(y)$, that is, $y \in A$.

Now let $x, y \in A$ and $x \perp y$. By the orthogonal additivity of $f, f(x+y)=f(x)+f(y)=$ $x+y$, and so, $x+y \in A$. Thus, $A$ is a lateral ideal.

So, it remains to prove that $A$ is laterally closed. Let $\left(x_{\alpha}\right)$ be a net in $A$ with $x_{\alpha} \sqsubseteq x_{\beta}$ for $\alpha \leq \beta$ and $x=\sqsubseteq-\sup _{\alpha} x_{\alpha}$. By [2], $x_{\alpha} \sqsubseteq x$ for all $\alpha$ and so, $x_{\alpha} \stackrel{\ell \uparrow}{\longrightarrow} x$. By the up-laterally continuity of $f$ (see (2)), we obtain $x_{\alpha}=f\left(x_{\alpha}\right) \stackrel{\ell \uparrow}{\longrightarrow} f(x)$. The above conditions imply $f(x)=x$, that is, $x \in A$.

(4) follows from (3) and Remark 1.

\section{THE PRINCIPAL LATERAL BAND PROJECTION}

Obviously, both lateral ideal and lateral band, generated by a singleton $\{e\}$, where $e \in E$, coincide with the set $\mathfrak{F}_{e}$ of all fragments of $e$, which we call the principal lateral ideal and the principal lateral band generated by $e$.

In this section, we describe lateral band projections onto principal lateral bands in a Riesz space with the principal projection property.

Theorem 4. Let $E$ be a Riesz space with the principal projection property. Then for every e $\in E$ the function $Q_{e}: E \rightarrow E$ defined by setting

$$
Q_{e} x=x \cap e \text { for all } x \in E
$$

is a lateral retraction of $E$ onto the principal lateral band $\mathfrak{F}_{e}$.

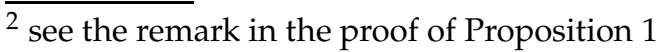


Proof. Observe that the operator $Q_{e}$ is well defined by (2), because every finite subset of a Riesz space with the principal projection property has a lateral infimum [6, Theorem 3.13].

We prove that $Q_{e}$ satisfies all items of Definition 2. Items (i) and (iii) are obvious.

(ii) Let $x, y \in E \backslash\{0\}$ and $x \perp y$. Observe that $B_{x+y}=B_{x} \oplus B_{y}$, and hence, every $z \in B_{x+y}$ has an expansion

$$
z=P_{x} z+P_{y} z
$$

We prove that

$$
P_{x}((x+y) \cap e)=x \cap e, \quad P_{y}((x+y) \cap e)=y \cap e .
$$

Since the band projection $P_{x}$ preserves disjointness, $P_{x}$ is lateral order preserving, that is,

$$
(\forall u, v \in E) u \sqsubseteq v \Rightarrow P_{x} u \sqsubseteq P_{x} v
$$

Indeed, $u \sqsubseteq v$ means $u \perp(v-u)$, which yields $P_{x} u \perp P_{x}(v-u)=P_{x} v-P_{x} u$, that is, $P_{x} u \sqsubseteq P_{x} v$.

Hence by (5),

$$
P_{x}((x+y) \cap e) \sqsubseteq P_{x}(x+y)=x .
$$

On the other hand, by the obvious relation $P_{x} w \sqsubseteq w$ for all $w \in E$,

$$
P_{x}((x+y) \cap e) \sqsubseteq(x+y) \cap e \sqsubseteq e .
$$

By (6) and (7),

$$
P_{x}((x+y) \cap e) \sqsubseteq x \cap e .
$$

Observe that $|x \cap e| \leq n|x|$ for all $n \in \mathbb{N}$, and hence, by [1, Theorem 1.47],

$$
P_{x}(x \cap e)^{+}=\bigvee_{n=1}^{\infty}\left((x \cap e)^{+} \wedge n|x|\right)=(x \cap e)^{+}
$$

and analogously, $P_{x}(x \cap e)^{-}=(x \cap e)^{-}$. Therefore,

$$
x \cap e=P_{x}(x \cap e) \stackrel{\text { by }(5)}{\sqsubseteq} P_{x}((x+y) \cap e) .
$$

By (8) and (9), the first part of (4) is proved. The second part is proved analogously. Finally, we obtain

$$
(x+y) \cap e \stackrel{\text { by (3) }}{=} P_{x}((x+y) \cap e)+P_{y}((x+y) \cap e) \stackrel{\text { by (4) }}{=}(x \cap e)+(y \cap e),
$$

and the orthogonal additivity of $Q_{e}$ is proved.

We do not know if the Dedekind complete assumption in item (4) of Theorem 3 is essential.

Problem 1. Is there a Riesz space $E$ and a lateral band $A$ of $E$ which is not a projection lateral band (equivalently, which is not a lateral retract)? 


\section{REFERENCES}

[1] Aliprantis C.D., Burkinshaw O. Positive Operators. Springer, Dordrecht, 2006.

[2] Gumenchuk A.I. Lateral continuity and orthogonally additive operators. Carpathian Math. Publ. 2015, 7 (1), 49-56. doi:10.15330/cmp.7.1.49-56

[3] Gumenchuk A.I., Pliev M.A., Popov M.M. Extensions of orthogonally additive operators. Mat. Studii 2014, 41 (2), 214-219.

[4] Mazón J.M., Segura de León S. Order bounded ortogonally additive operators. Rev. Roumaine Math. Pures Appl. 1990, 35 (4), 329-353.

[5] Mazón J.M., Segura de León S. Uryson operators. Rev. Roumaine Math. Pures Appl. 1990, 35 (4), $431-449$.

[6] Mykhaylyuk V., Pliev M., Popov M. The lateral order on Riesz spaces and orthogonally additive operators. Preprint.

[7] Pliev M., Popov M. Narrow orthogonally additive operators. Positivity 2014, 18, 641-667. doi:10.1134/S0037446617010177

[8] Pliev M., Popov M. On extension of abstract Uryson operators. Sib. Mat. Zh. 2016, 57 (3), $700-708$ (in Russian).

[9] Pliev M., Ramdane K. Order unbounded orthogonally additive operators in vector lattices. Mediterr. J. Math. 2018, 15 (2), Art. 55, 20 pp. doi:10.1007/s00009-018-1100-5

Received 01.03.2020

Каміньська А., Красікова І., Попов М. Проекційні латеральні смуги та латеральні ретракти // Карпатські матем. публ. - 2020. - Т.12, №2. - С. 333-339.

Проекційну латеральну смугу $G$ у векторній гратці $E$ визначають як латеральну смугу, яка $\epsilon$ образом деякого ортогонально адитивного проектора $Q: E \rightarrow E$, що має таку властивість: $Q(x) \in$ фррагментом елемента $x$ лля всіх $x \in E$; такий проектор називають латеральною ретракцією гратки $E$ на $G$ (доводиться єдиність такого проектора). Ми досліджуємо властивості латеральних ретрактів, тобто образів латеральних ретракцій, і описуємо латеральні ретракції на головні проекційні латеральні смуги (тобто латеральні смуги, породжені окремими елементами) у векторній гратці з головною проективною властивістю. Крім того, ми доводимо, що кожний латеральний ретракт є латеральною смугою, а кожна латеральна смуга у порядково повній векторній гратці є проекційною латеральною смугою.

Ключові слова $і$ фрази: ортогонально адитивний оператор, векторна гратка, латеральна смуга. 